\title{
Lessons from patients' journeys
}

\author{
Peter Lapsley, patient editor at the $B M J$, reflects on the lessons that have emerged from the first \\ 100 articles in the Patient's Journey series
}

\author{
Peter Lapsley patient editor, BMJ \\ BMA House, London WC1H 9JP, UK
}

Over the past seven or eight years, I have been helping patients to get their stories published, obtaining doctors' perspectives on them, and, with colleagues, developing the guidelines for drafting patients' journeys, hoping that these might increase the usefulness of such articles to doctors (box).

I describe here some of the wide range of lessons that have emerged from the patients' journeys that we have published.

\section{Certainty and uncertainty}

We have repeatedly learnt that although doctors are comfortable with uncertainty, patients are far less so. They want prompt and accurate diagnoses, taking the view that a condition cannot be treated effectively if the doctor doesn't know what it is. And they need a label to explain their conditions to family and friends. Of the numerous examples of this, one of the more recent was an account of trachea-oesophageal atresia. ${ }^{1}$

Although medicine is a constantly evolving discipline, many patients fail to recognise that doctors don't have a solution for every problem. Patients also tend to regard their conditions as unique, as if their doctor doesn't see dozens of patients with similar problems each week. Doctors may easily dismiss apparently common conditions, and this is demoralising for patients and potentially dangerous - dismissiveness can lead to misdiagnosis, or a missed diagnosis, of a serious condition.

\section{Time to diagnosis}

Some patients may be as responsible as doctors for delayed diagnoses, often because the condition "creeps up on them," becoming gradually worse. By the time they seek medical advice the disease may be far advanced-and possibly terminal.

Quite often, too, patients are either embarrassed by their symptoms or reluctant to "waste the doctor's time." Doctors see embarrassing conditions all the time, but their patients do not, and doctors need to be sensitive to their feelings. ${ }^{2}$ Similarly, it doesn't usually help patients to be told that their problems are trivial or routine: they will have consulted a doctor because they are concerned. They need their worries allayed, not dismissed.
Doctors too can be responsible for delays, taking too long to correctly diagnose a condition. Attachment to a "working diagnosis" long after the appearance of new signs and symptoms is a particular trap, as the general practitioner who was managing a case of ovarian cancer as irritable bowel syndrome admitted. ${ }^{3}$

One of the most important lessons from the series has been the importance of considering apparently unlikely diagnoses as well as the most obvious ones, a lesson eloquently expressed in "Sir Karl Popper, swans, and the general practitioner." ${ }^{4}$ Perhaps the least forgivable delay occurred in a case of Klinefelter's syndrome: the condition was correctly diagnosed when the patient was 14 years old but the diagnosis was then "lost" and he went untreated for 46 years until the condition was correctly diagnosed again. ${ }^{5}$

More worrying, though, are cases of rare conditions, where uncertainty should have led to early referral for a specialist opinion. That is one reason why rare conditions feature more often in the Patient's Journey series than in the rest of the Practice section of the $B M J$ : we hope that these articles may trigger recollection when, very occasionally, a patient presents with one. The case of xeroderma pigmentosum described in Alex Webb's account ${ }^{6}$ would have not have taken so long to diagnose if his doctors had taken the message of Karl Popper's swans to heart.

\section{Social, physiological, and psychological consequences}

Every patient's journey explains the consequences for the patient, their family and friends, and their relationships with their health professionals.

Unsurprisingly, several of them have been gruelling - to me as a layman, at least. The account of the mesothelioma from which the author was to die was particularly so, $^{7}$ as was the account of obstetric fistula, which occurs mostly in developing countries and usually leads to women being disowned by their husbands and families and left to beg in the street. ${ }^{8}$ 


\section{Our guidance to authors of patients' journeys}

To some extent, our published guidance to authors of patients' journeys dictates the sort of articles we get. The following list is not exhaustive, but our advice suggests that the articles should demonstrate one or more of the following lessons.

- Some doctors take too long to arrive at a correct diagnosis. By reading about patients' journeys, other doctors might diagnose the condition sooner, which would benefit their patients

- Some aspects of conditions and diseases are very important to patients but doctors may be unaware of these aspects

- Evidence based interventions may exist that could have profound effects on patients' lives and of which doctors should be more aware

We have seen these lessons repeated in so many patients' journeys.

We are always open to suggestions for improving our series

Almost as saddening have been the accounts of the burdens placed on carers by chronic, terminal conditions, vividly demonstrated in husbands' accounts of the deaths of their wives from motor neurone disease ${ }^{9}$ and dementia. ${ }^{10}$

Doctors mostly see patients for only a few minutes occasionally and focus during that time on the illness itself, so it's easy for them to forget about the effects that the illness is having on the patient's everyday life. Indeed, rarely does a doctor know what a patient does on leaving the surgery, the things that interest and preoccupy them, their needs and aspirations, and their capabilities and limitations. Key to the successful treatment of a condition-or of multiple conditions-is to "see the person in the patient." 11

Another lesson to have emerged from the articles is how modest adjustments could vastly improve patients' experience of treatment. Irritating difficulties abound: with travel; long waits for appointments in both primary and secondary care; long waits between follow-up appointments. These last two, in particular, tie in with the observation that doctors know little about how patients behave when away from their surgeries. They also fuel the public perception that the health service is oblivious to the fact that patients have their own lives to lead.

\section{New or underused interventions}

This is one of the aspects that causes us a fair amount of soul searching. We don't see Patient's Journey articles as a means of providing opportunities for doctors to promote new treatments for which there is little or no evidence base for efficacy and safety. Some submissions have been attempts to "drum up business" for a new intervention. If a treatment is believed to work in a particular condition, the $B M J$ would need a proper study to provide robust evidence of that. A promising result in a single patient is scarcely admissible as evidence.

Some of the "underused" interventions are organisational, rather than clinical, an excellent example being seen in an account of a patient's two hip replacements at the same hospital, ${ }^{12} 12$ years apart, during which time postoperative treatment had been greatly improved by the development of a team based approach. Another underused intervention involves the benefits of continuity of care and the development of good working relationships between patients and health professionals, demonstrated clearly in the patient's journey about psoriasis. ${ }^{13}$

Underused interventions include such life enhancing resources as skin camouflage, shown vividly in the patient's journey about facial disfigurement. ${ }^{14}$ And the catalogue of aids to mobility and daily living given in one patient's account of his road to recovery from spinal cord injury ${ }^{15}$ should serve to remind doctors of resources they either knew nothing of or about which they may have forgotten.

\section{And now for the good news}

One lesson that shines through almost all the patients' journeys is the admiration and respect that patients have for clinicians. This is not because they know that their treating physicians will be invited to write about their cases; it is genuine and honest and reinforces the findings of surveys that have shown repeatedly that the great majority of patients who have used the NHS recently think it is excellent.

From a more personal point of view, as a non-doctor, I have been greatly surprised by the sheer number of medical conditions and interventions of which clinicians need to have at least some understanding. Even though we have published about 100 patients' journeys so far, we receive a constant stream of new offerings, usually instigated by doctors. Indeed, we currently have more than 10 articles accepted and awaiting publication and many more in the pipeline.

When the series began in 2006, the articles were written solely by patients and tended to be better suited to the colour supplements of Sunday papers than to the $B M J$, the role of which is to help doctors make better decisions. Recognising this, we introduced the "doctors' perspectives" boxes, usually written by the patients' own treating physicians, giving a brief overview of the condition or intervention, saying what he or she learnt from this particular case and suggesting lessons the BMJ's readers might take from it. That seems to have worked well. It should come as no surprise, though, that the best patients' journeys are those written by doctors who have themselves been patients. They know what they have and have not been taught and can see the lessons for doctors in the stories they have to tell; lay patients are rarely so well informed.

I have been impressed, too, by doctors' willingness to learn from patients' stories. Where things have gone wrong, doctors have almost always said so. Perhaps the most dramatic examples are a patient's account of ovarian cancer, ${ }^{3}$ which led to an in-practice review (in which she was included) and to a general practitioner's suggestion that she write the case up for the Patient's Journey series; and a woman's gruelling account of being conscious but paralysed through an emergency caesarean section $^{16}$; she and her anaesthetist have since discussed in detail what happened and become friends.

In a few cases, the patients' doctors have sought to correct what they regard as inaccuracies in patients' stories, rather than exploring the misunderstandings. Far better to leave the inaccuracy in and say in the doctor's perspective box, "Is that what the patient thought I meant? I wonder why."

Reassuringly few healthcare units have reacted badly to what's been written about them, instead trying to resolve the patient's concerns. It points up one of the biggest lessons of all: that doctors and patients can view aspects of the same "treatment" very differently. There is no privileged vantage point from which to decide who is right and who is wrong. This leads us to what may be the most significant lesson of all, the importance of good doctor-patient communication, which needs to be as 
jargon-free as possible, be empathetic, and bridge educational, social, and age related boundaries. It is good that British medical schools now place so much emphasis on this.

Competing interests: The author declares no support from any organisation for the submitted work; no financial relationships with any organisations that might have an interest in the submitted work in the previous three years, no other relationships or activities that could appear to have influenced the submitted work.

Provenance and peer review: Commissioned; not externally peer reviewed.

Love C, Morice AH. After repair of trachea-oesophageal atresia. BMJ 2012;344:e3517. Kelly S. Ulcerative colitis. BMJ 2012;344:e2947.

Reeve G, Mackay-Thomas S. The invisible worm: ovarian cancer. BMJ 2009;338:b2072.
4 Berghmans R, Schouten HC. Sir Karl Popper, swans, and the general practitioner. BMJ 2011;343:d5469.

5 Anonymous, Bhartia M, Ramachandran S. Klinefelter's syndrome. BMJ 2012;345:e6938.

6 Webb S, Woollons A. Xeroderma pigmentosum. BMJ 2008;336:444.

7 Sweeney K, Toy L, Cornwell J. Mesothelioma. BMJ 2009;339:b2862.

Aliyu F, Esegbona G. Living with obstetric fistula. BMJ 2011;342:d2881.

Sackett B, Sakel M. Motor neurone disease BMJ 2011;342: 1661 .

10 Dartington T, Sampson EL. Dying from dementia. BMJ 2008:337:a1712.

11 Goodrich J, Cornwell J. Seeing the person in the patient. 2008. www.kingsfund.org.uk/ sites/files/kf/Seeing-the-person-in-the-patient-The-Point-of-Care-review-paper-GoodrichCornwell-Kings-Fund-December-2008.pdf.

12 Tomlinson P, Stranks GJ. Two hip replacements. BMJ 2010;340:c150

13 Jobling R, Norris P. Psoriasis. BMJ 2007;334:953.

14 Saul K, Thistlethwaite JE. Facial disfigurement. BMJ 2011;343:d5203.

15 Sonksen P, Hillier S. Spinal cord injury. BMJ 2010;340:b5204.

16 Aaen A-M, Møller K. Through and beyond anaesthesia awareness. BMJ 2010;341:c3669.

Cite this as: BMJ 2013;346:f1988

(c) BMJ Publishing Group Ltd 2013 